5. Conclusion. The methods presented above have been applied to more complicated frames and readily give the required solutions for collapse design under constant or varying loads. For shakedown design, the iterative numerical method converges fairly rapidly. It may be found easier for highly redundant frames to obtain a new elastic solution at each stage using the numerical values obtained from the previous analysis, since an analysis with numerically unspecified flexural rigidities is extremely tedious. For all three types of design, the introduction at an early stage of the numerical values of the loads simplifies the work greatly, since it will be found that a large proportion of the inequalities generated become redundant and can be ignored.

Only examples of concentrated loads on straight members of uniform cross-section between joints have been examined, making it possible to pick by inspection the critical cross-sections. However, the basic ideas are not altered by the introduction of other variables; the analysis will be more complicated, but aids to calculation may be introduced which leave the basic problem unchanged.

\title{
THE METHOD OF CHARACTERISTICS APPLIED TO PROBLEMS OF STEADY MOTION IN PLANE PLASTIC STRESS*
}

\author{
By P. G. HODGE, JR. (University of California at Los Angeles)
}

A method is outlined for obtaining the stress, strain, and thickness distribution in a thin sheet which is strained plastically in its plane. For the particular case of steady motion, a method is given for obtaining directly the final solution to certain types of boundary value problems. A step by step procedure is indicated for the general case of non-steady motion.

1. Introduction. This paper is concerned with the stress and strain distribution in a thin sheet which is strained plastically in its plane, under conditions of plane stress. It will be shown that three types of problems may be distinguished. In certain special cases the stress distribution may be found independently of the velocity or thickness by solving three equations in as many unknowns. For general steady motion problems it will be necessary to solve six equations simultaneously for three stress components, two velocity components, and the thickness. These equations will be stated in Sec. 2, and reduced to a system of five first order, quasi-linear differential equations under the assumption of initial isotropy. For suitable boundary conditions, it will be possible to find the final stress, strain, and thickness distribution of the material directly, using the method of characteristics. The details of this method of solution will be described in Secs. 3 and 4. Finally, in Sec. 5, a step-by-step procedure for solving problems of non-steady motion will be briefly indicated.

2. Basic equations. Let the sheet be referred to a set of Cartesian axes such that the $x, y$ plane coincides with the middle surface, and let $z= \pm \frac{1}{2} h(x, y)$ be the equations of the bounding surfaces. Under the assumptions of generalized plane stress, the only non-vanishing averaged stress components are $\sigma_{x}, \sigma_{y}$, and $\tau_{x y}$. These components must satisfy the equations of equilibrium

\footnotetext{
*Received March 29, 1950.
} 


$$
\begin{aligned}
& \frac{\partial}{\partial x}\left(h \sigma_{x}\right)+\frac{\partial}{\partial y}\left(h \tau_{x y}\right)=X, \\
& \frac{\partial}{\partial x}\left(h \tau_{x y}\right)+\frac{\partial}{\partial y}\left(h \sigma_{y}\right)=Y .
\end{aligned}
$$

Here $X$ and $Y$ are the components of body force in the $x$ and $y$ directions, respectively. They may be functions of $x, y$, and $h$.

In addition, if the material is to behave plastically, the stress components must satisfy a yield condition which may be written in the form

$$
F\left(\sigma_{x}, \sigma_{y}, \tau_{x y}\right)=0 .
$$

Strictly speaking, Eq. 1(c) must be satisfied by the actual stress components. However, if the variation of the stress components across the thickness is not too great, it will be satisfied by the averaged stress components to a high degree of accuracy.

In the particular case where $h$ is known, and where the boundary conditions in stresses are sufficient to set a fully plastic problem, Eqs. 1(a-c) may be solved directly for the stresses with no reference to the velocities. ${ }^{1}$ However, in general neither of these conditions will be satisfied, and it is necessary to consider the stress-strain relations. According to the "plastic potential" stress-strain law,

$$
\frac{\partial u / \partial x}{\partial F / \partial \sigma_{x}}=\frac{\partial v / \partial y}{\partial F / \partial \sigma_{y}}=\frac{\partial u / \partial y+\partial v / \partial x}{\partial F / \partial \tau_{x y}} .
$$

Since $F$ is a known function, Eqs. 1(d) provide two additional equations, but introduce two additional functions $u$ and $v$, the averaged velocity components in the $x$ and $y$ directions respectively. The final necessary equation is obtained from the assumption that the plastic material is incompressible. For steady flow, this condition may be written in the form ${ }^{3}$

$$
\frac{\partial}{\partial x}(h u)+\frac{\partial}{\partial y}(h v)=0 .
$$

Equations 1(a-e) are a set of six equations for the six unknown functions $\sigma_{x}, \sigma_{y}, \tau_{x y}$, $h, u$, and $v$.

It will prove convenient to make the substitutions

$$
\left.\begin{array}{ll}
\sigma_{x}=2 k[\omega+\chi \sin 2 \theta], & \tau_{x y}=-2 k \chi \cos 2 \theta, \\
\sigma_{y}=2 k[\omega-\chi \sin 2 \theta], & H=\log h .
\end{array}\right\}
$$

The function $\theta$ may be interpreted as the angle between a fixed direction and a principal direction (see, for instance, Ref. footnote 1), so that if the yield condition is isotropic, it may be written

$$
F\left(\sigma_{x}, \sigma_{y}, \tau_{x y}\right)=\chi-f(\omega)=0 .
$$

${ }^{1}$ This has been done by the author in another paper. P. G. Hodge, Jr., Yield conditions in plane plastic stress, to be published in the J. Math. and Physics.

${ }^{2}$ R. v. Mises, Mechanik der plastischen Formaenderung von Kristallen, ZS. angew. Math. Mech., 8, 161-185 (1928).

${ }^{3}$ R. Hill, Plastic distortion of non-uniform sheets, Phil. Mag. (7) 40, 971-983 (1949). 
The substitution of Eqs. 2 into Eqs. 1 leads to the five equations $\left(1+f^{\prime} \sin 2 \theta\right) \omega_{x}-f^{\prime} \cos 2 \theta \omega_{y}+2 f\left(\cos 2 \theta \theta_{x}+\sin 2 \theta \theta_{y}\right)$

$$
+(\omega+f \sin 2 \theta) H_{x}-f \cos 2 \theta H_{\nu}=X,
$$

$-f^{\prime} \cos 2 \theta \omega_{x}+\left(1-f^{\prime} \sin 2 \theta\right) \omega_{y}+2 f\left(\sin 2 \theta \theta_{x}-\cos 2 \theta \theta_{y}\right)$

$$
\begin{gathered}
-f \cos 2 \theta H_{x}+(\omega-f \sin 2 \theta) H_{\nu}=Y, \\
u_{x}+v_{\nu}+u H_{x}+v H_{\nu}=0, \\
-2 \cos 2 \theta u_{x}+\left(f^{\prime}-\sin 2 \theta\right)\left(u_{\nu}+v_{x}\right)=0, \\
\left(f^{\prime}+\sin 2 \theta\right)\left(u_{\nu}+v_{x}\right)-2 \cos 2 \theta v_{\nu}=0,
\end{gathered}
$$

where subscripts now indicate differentiation. Equations $3(\mathrm{a}-\mathrm{e})$ are a set of 5 quasilinear first order equations for the 5 unknown functions $\omega, \theta, u, v, H$. Once these quantities have been determined, the stress components are easily found from Eqs. 2.

3. Characteristic equations. The characteristics of Eqs. 3 may be defined as those curves across which it is possible for the derivatives of the unknown functions to exhibit finite jump discontinuities, the functions themselves being continuous. Let $x=x(s)$, $y=y(s)$ be the parametric equations of a characteristic curve, and let $\omega, \theta, u, v$ and $H$ be given along the curve as functions of the parameter $s$. Then, along the curve,

$$
\left.\begin{array}{c}
u_{x} d x+u_{\nu} d y=d u, \quad v_{x} d x+v_{\nu} d y=d v, \\
\omega_{x} d x+\omega_{y} d y=d \omega, \quad \theta_{x} d x+\theta_{\nu} d y=d \theta, \\
H_{x} d x+H_{\nu} d y=d H .
\end{array}\right\}
$$

Equations 3 and 4 may be regarded as a set of 10 linear algebraic equations for the 10 unknowns $\omega_{x}, \omega_{y}, \theta_{x}, \cdots, H_{y}$. In general they will possess a unique solution, so that a discontinuity in these unknown derivatives will not be permitted. An exception can occur only if the slope of the curve, $d y / d x$, is such that the determinant of the coefficients $D_{0}$ vanishes. By means of Laplace's expansion, ${ }^{4}$ the $10 \times 10$ determinant of $D_{0}$ may be reduced to the product of three determinants:

where

$$
D_{0}=D_{0}^{\prime} \cdot D_{0}^{\prime \prime} \cdot D_{0}^{\prime \prime \prime},
$$

$$
D_{0}^{\prime}=\left|\begin{array}{cccc}
1+f^{\prime} \sin 2 \theta & -f^{\prime} \cos 2 \theta & 2 f \cos 2 \theta & 2 f \sin 2 \theta \\
-f^{\prime} \cos 2 \theta & 1-f^{\prime} \sin 2 \theta & 2 f \sin 2 \theta & -2 f \cos 2 \theta \\
d x & d y & 0 & 0 \\
0 & 0 & d x & d y
\end{array}\right|,
$$

4See, for instance, A. C. Aitken, Determinants and matrices, Oliver and Boyd, London, 1939. 
and

$$
D_{0}^{\prime \prime \prime}=\left|\begin{array}{cccc}
-2 \cos 2 \theta & f^{\prime}-\sin 2 \theta & f^{\prime}-\sin 2 \theta & 0 \\
0 & f^{\prime}+\sin 2 \theta & f^{\prime}+\sin 2 \theta & -2 \cos 2 \theta \\
d x & d y & 0 & 0 \\
0 & 0 & d x & d y
\end{array}\right| .
$$

$D_{0}^{\prime}$ and $D_{0}^{\prime \prime \prime}$ each vanish along either of the curves

$$
d y / d x=-\cot \alpha, \quad d y / d x=\tan \beta,
$$

where the substitutions

$$
f^{\prime}=\sin 2 \psi(|\psi|<\pi / \psi), \quad \alpha=\theta+x, \quad \beta=\theta-x
$$

have been made. Obviously these characteristics are real only if $\left|f^{\prime}\right| \leq 1$; n the remainder of this paper it will be assumed that $\left|f^{\prime}\right|$ is actually less than one. ${ }^{5}$

Each of the curves 5(a) represent a multiple characteristic. The final family of characteristics is obtained by setting $D_{0}^{\prime \prime}=0$ :

$$
d y / d x=v / u \text {. }
$$

The curves 5(a) are called the first and second characteristics, respectively, and the curves $5(\mathrm{~b})$ are called the streamlines.

4. Conditions along the characteristics. The problem of finding conditions which must be satisfied along the characteristics is complicated by the existence of multiple characteristics. It will prove convenient to use one method for Eqs. 3(d,e) and find the velocity conditions along the characteristics. The velocity derivatives will then be regarded as known, and a different method applied to yield the stress relations along the characteristics and the relations along the streamlines.

Consider the characteristic curves (Eqs. 5a) as curvilinear coordinates $\xi=$ const., $\eta=$ const., where $\xi$ and $\eta$ are functions of $x$ and $y$. It is easily shown that the relations

$$
\eta_{x} \sin \alpha+\eta_{y} \cos \alpha=0, \quad \xi_{x} \cos \beta+\xi_{y} \sin \beta=0,
$$

must be valid along a first and second characteristic, respectively.

The substitution of Eqs. 6, together with the relations $u_{x}=u_{\xi} \xi_{x}+u_{\eta} \eta_{x}$, etc. into Eqs. 3(d,e) leads to the equations

$$
\left.\begin{array}{l}
\xi_{x} \sin \beta\left[u_{\xi} \sin \alpha-v_{\xi} \cos \alpha\right]-\eta_{x} \cos \alpha\left[u_{\eta} \cos \beta+v_{\eta} \sin \beta\right]=0, \\
\xi_{x} \cos \alpha \cos ^{2} \beta\left[u_{\xi} \sin \alpha-v_{\xi} \cos \alpha\right]-\eta_{x} \sin \beta \sin ^{2} \alpha\left[u_{\eta} \cos \beta+v_{\eta} \sin \beta\right]=0 .
\end{array}\right\}
$$

If Eqs. 7 are regarded as simultaneous linear equations for the expressions in square brackets, it is seen that the only solution is the trivial one. In view of the definitions of $\xi$ and $\eta$, this may be written as

$$
d u \sin \alpha-d v \cos \alpha=0
$$

${ }^{5}$ The meaning of this restriction has been discussed by the author (see footnote 1 ) and by $\mathrm{R}$. Hill (see footnote 3 ). 
along a first characteristic, and

$$
d u \cos \beta+d v \sin \beta=0
$$

along a second characteristic.

To find the relations between $\omega, \theta$, and $H$ along the characteristics and streamlines, let us assume that $u$ and $v$ and their derivatives are known from Eqs. 8(a, b) and consider Eqs. 3(a-c) as a set of three equations for the three unknowns $\omega, \theta$, and $H$. The characteristics of these three equations will be those curves for which the determinant of the coefficients of $\omega_{x}, \cdots, H_{y}$ vanishes. Let this determinant be denoted by $\Delta_{0}$, and let $\Delta_{k}$ be the determinant formed by replacing the $k$ th column of $\Delta_{0}$ by the inhomogeneous terms (i.e., the terms not containing $\omega_{x}, \cdots, H_{y}$ ) of Eqs. 3(a-c) and 4(b). In order for the derivatives $\omega_{x}, \cdots, H_{y}$ to be able to exhibit finite discontinuities across the characteristics, it is necessary for all of the determinants $\Delta_{0}, \Delta_{1}, \cdots, \Delta_{6}$ to vanish. The first condition leads to the three curves given by Eqs. 5, of course, and the remaining conditions lead to the relations

$$
\begin{gathered}
(u \cos \alpha+v \sin \alpha)[2 f d \theta-\cos 2 \psi d \omega-(X \sin \beta-Y \cos \beta)(d y / \cos \alpha)] \\
=[(u \cos \beta+v \sin \beta) \omega+(-u \sin \alpha+v \cos \alpha) f] d H \\
\quad+\left(u_{x}+v_{u}\right)(f-\omega \sin 2 \psi)(d y / \cos \alpha)
\end{gathered}
$$

along a first characteristic,

$$
\begin{gathered}
(u \sin \beta-v \cos \beta)[2 f d \theta+\cos 2 \psi d \omega-(X \cos \alpha+Y \sin \alpha)(d y / \sin \beta)] \\
=[(-u \sin \alpha+v \cos \alpha) \omega+(u \cos \beta+v \sin \beta) f] d H \\
\quad+\left(u_{x}+v_{y}\right)(f-\omega \sin 2 \psi)(d y / \sin \beta)
\end{gathered}
$$

along a second characteristic, and

$$
\left(u_{x}+v_{y}\right) d y+v d H=0
$$

along a streamline.

5. Conclusion. Equations 5 and 8 may be replaced by finite difference equations and used to obtain numerical solutions of boundary value problems. There are a great number of possible such problems, corresponding to various combinations of the Cauchy and Riemann problems for a system of two hyperbolic equations. The details of the method may be readily adopted from corresponding treatments in compressible flow. ${ }^{6}$

For the general case of non-steady motion, it is necessary to first solve the problem at the instant $t_{0}$ when plastic flow begins. Since $H$ is an initially known function, this involves solving Eqs. $3(\mathrm{a}, \mathrm{b}, \mathrm{d}, \mathrm{e})$ for $\omega, \theta, u$, and $v$. This problem is similar to the one discussed in the preceding sections, and, in fact, $(5 \mathrm{a})$ and $8(\mathrm{a}, \mathrm{b}, \mathrm{c}, \mathrm{d})$ may be used, since they are not dependent upon the motion being steady. The right hand side of

${ }^{6}$ See, for instance, W. Tollmien, Stationaere ebene und rotationssymmetrische Uberschallstroemungen, Technische Hochschule Dresden, 1940. This work has been translated by the Air Materiel Command, Wright Field, Dayton, Ohio as translation A9-T-1. Contract W33-038-ac-15004 (16351), Brown University, Providence, Rhode Island. 
Eq. 3(c) must now be replaced by $-H_{t}$, and, since the left side of Eq. 3(c) is known at $t=t_{0}, H_{t}\left(t_{0}\right)$ is known throughout the field. A finite difference approximation then yields $H\left(t_{0}+\Delta t\right)$ throughout the field, so that the process may be repeated. Thus a step-by-step solution may be obtained for all values of $t$.

\title{
A NOTE ON THE PRINCIPAL FREQUENCY OF A TRIANGULAR MEMBRANE* '
}

\author{
By G. PÓLYA (Stanford University)
}

The principal frequency of a membrane of triangular shape is exactly known in two simple cases: for the $45^{\circ}, 45^{\circ}, 90^{\circ}$ and the $60^{\circ}, 60^{\circ}, 60^{\circ}$ triangles. ${ }^{2}$ As will be shown in this note, an exact solution of comparable simplicity exists also for the $30^{\circ}, 60^{\circ}, 90^{\circ}$ triangle-a result which, to the author's knowledge, has not been observed before.

The three lines the equations of which in rectangular coordinates $x, y$ are

$$
y=0, \quad x=a 3^{1 / 2} / 2, \quad y=x 3^{-1 / 2}
$$

delimit a triangle, one half of an equilateral triangle with side $a$. Define

$$
\begin{aligned}
u=\sin \pi( & \left(y-3^{-1 / 2} x\right) / a \cdot \sin \pi\left(y+3^{-1 / 2} x\right) / a \cdot \sin 2 \pi 3^{-1 / 2} x / a \\
\cdot & \sin \pi\left(y-3^{1 / 2} x\right) / 3 a \cdot \sin \pi\left(y+3^{1 / 2} x\right) / 3 a \cdot \sin 2 \pi y / 3 a
\end{aligned}
$$

By elementary transformations we find that

$$
\begin{aligned}
-32 u & =\cos 2 \pi\left(5 y+3^{1 / 2} x\right) / 3 a-\cos 2 \pi\left(4 y+3^{1 / 2} 2 x\right) / 3 a+\cos 2 \pi\left(y+3^{1 / 2} 3 x\right) / 3 a \\
& -\cos 2 \pi\left(5 y-3^{1 / 2} x\right) / 3 a+\cos 2 \pi\left(4 y-3^{1 / 2} 2 x\right) / 3 a-\cos 2 \pi\left(y-3^{1 / 2} 3 x\right) / 3 a .
\end{aligned}
$$

We see from (3) that $u$ satisfies the equation

$$
u_{x x}+u_{y y}+9^{-1} 112 \pi^{2} a^{-2} u=0,
$$

from (2) or (3) that $u$ vanishes along the lines (1), and from (2) that $u$ does not vanish in the interior of the triangle between the lines (1). Therefore, $u$ represents the principal mode.

The well known solution for the two other triangles mentioned at the beginning can be presented in a strictly analogous form. Any of these three triangles, repeated by successive reflections, covers the whole plane without overlapping and there are no further triangles of this kind. Therefore, there are no other triangles for which the solution can be presented in a comparably simple form as a function of $x, y$ without singularity in the whole plane. The last remarks indicate also the heuristic reasoning which led to the solution (2).

*Received April 17, 1950.

'Sponsored by the Office of Naval Research.

${ }^{2}$ Rayleigh, The theory of sound, Dover Publications, New York, 1945, §199. 